(Russia, Cheboksary) has unique properties. The right to extract and use sapropel belongs to the sanatorium "Chuvashiyakurort" (Russia) [1]. The organic biostimulants of the sapropel mud (bitumen, fatty and humic acids, amino acids, hormones, lipids) have a pronounced anti-inflammatory effect on cartilage. High humidity $(87 \%)$ provides sapropel high thermal qualities.

Objectives: The aim of the research was to study the effectiveness of peloid therapy in patients with osteoarthritis of the knee joints at the II X-ray stage using the sapropel mud of Lake «Kogoyar».

Methods: The object of the study was 150 patients of the sanatorium "Chuvashiyakurort" aged from 55 to 62 years ( 89 women and 61 men). The patients were randomly divided into 2 groups. The first group (75 people) received basic therapy - chondroprotectors (Teraflex) and nonsteroidal anti-inflammatory drugs (NSAIDs) on demand. The second group (75 people) received basic therapy with the addition of peloid application. The peloid was used in the natural, minimally modified, undiluted form. The peloid therapy protocol: the peloid was heated to $50{ }^{\circ} \mathrm{C}$ before use and applied to the affected joint with a layer of $4-5 \mathrm{~cm}$ thick and wrapped with a warm cloth on top. The procedure took 30 minutes. Five threeday cycles of applications with one-day breaks were performed. At the end of the procedure, a patient was unraveled, the mud was removed from him and he took a warm shower. Then he had a rest for 30 to 40 minutes in the recreation room. Before the start of treatment and after its completion, a survey of patients was conducted with the fixation of pain on the VAS scale, the duration of morning stiffness, fatigue and decreased performance, general discomfort, dependence on taking NSAIDs.

Results: The analysis of the survey results revealed a decrease in VAS pain and morning stiffness in both groups. However, patients in the second group had a more pronounced reduction in the average VAS pain score ( $26 \%$ lower compared to the first group) and the duration of morning stiffness (20\% lower compared to the first group). In addition, the number of patients who indicated a decrease in dependence on taking NSAIDs was $28 \%$ higher than in the group that received only standard therapy.

Conclusion: Peloid therapy enabled to reduce the pain on the back, reduce the frequency of the need for NSAIDs, improve the condition of patients, which is the reflection of the positive clinical dynamics in patients with osteoarthritis of the knee joints, and contributed to the effectiveness of treatment.

REFERENCES:

[1] Sakharova A.S. Modern methods of medical rehabilitation in the practice of doctors of various specialties. Cheboksary: Publishing House of Chuvash State University, 2019: 110-115.

Disclosure of Interests: None declared.

DOI: 10.1136/annrheumdis-2021-eular.1256

\section{AB0590 ARTHRITIS OF THE KNEE AND BALANCE DISORDER}

W. Belhaj ${ }^{1}$, S. Miladi ${ }^{2}$, A. Fazaa ${ }^{2}$, M. Sellami ${ }^{2}$, K. Ouenniche ${ }^{1}$, L. Souebni ${ }^{1}$, S. Kassab ${ }^{1}$, S. Chekili ${ }^{1}$, K. Ben Abdelghani ${ }^{1}$, A. Laatar ${ }^{1} .{ }^{1}$ University of tunis el manar, Faculty of Medicine of Tunis, Mongi slim Hospital, Rheumatology Department, Tunis, Tunisia; ${ }^{2}$ University of Tunis el manar, Faculty of Medicine of Tunis, Mongi slim Hospital, Rheumatology Department, TUNIS, Tunisia

Background: Knee osteoarthritis has been identified as a risk factor for falls and balance disorders. Several clinical scales have been put in place to assess this risk.

Objectives: The aim of this work was to assess the risk of falls in patients followed for osteoarthritis of the knee.

Methods: A prospective study was conducted over a period of 4 weeks. Sixty-six patients followed for knee osteoarthritis at different stages of the disease were included into this work. Epidemiological data, physical examination data and $x$-ray data were collected. The impact of knee osteoarthritis was evaluated by the Lequesne and WOMAC indices. The patients were asked about the history of a fall since the onset of knee osteoarthritis and they had the Tinetti test where a score less than 20 reflects a very high risk of falling, from 20 to 23 significant risk, from 24 to 27 moderate risk and 28 means a normal test.

Results: In the studied population, the majority of patients were women (87\%) with an average age of 55 years and an average body mass index of $29 \mathrm{~kg} /$ m2. The most common comorbidities were high blood pressure $(61 \%)$, diabetes $(38 \%)$ and osteoporosis (36\%). Sixty-nine percent of patients took more than two oral treatments. The average duration of development of osteoarthritis of the knee was 9 years. The most common radiological stage was stage $1(58 \%)$ followed by stage 2 (31\%). The site was femorotibial in $88 \%$ of cases, patellofemoral in $4 \%$ of cases and bi or tri compartmental in $8 \%$ of cases. The arthritis most often affected both knees: $86 \%$ of cases. The average of the Lequesne index was 11. The mean WOMAC index was 12 in pain, 1.5 in stiffness and 48 in dysfunction. Patients who had a normal Tinetti score represented $39 \%$ of the studied population, those who had a score from 24 to 27 were $35 \%$ of the studied population, those who had a score between 20 and 23 were $14 \%$ of the studied population, and those who had a score below 20 were $12 \%$ of the studied population.
Patients reporting a history of one or multiple falls were $19 \%$. Among these patients $93 \%$ were women and $88 \%$ were hypertensive under antihypertensive treatment. The average age of these patients was 61 years. The Tinetti test revealed in this group of patients that $40 \%$ had a very high risk of falling, $48 \%$ had a high risk of falling, $10 \%$ had a moderate risk and $2 \%$ had a normal Tinetti test. These patients had been followed for knee osteoarthritis for an average of 10 years. The average Lequesne index was 12.5. The average WOMAC index was 12 for pain, 2 for stiffness and 55 for dysfunction.

In the following Table 1 we summarized the different stages of risk of falling according to the Tinetti score according to the epidemiological data and the characteristics of knee osteoarthritis:

\begin{tabular}{lllll}
\hline Tinetti score & Less than 20 & From 20 to 23 & From 24 to 27 & Equal to 28 \\
\hline Average age (years) & 66 & 65 & 56 & 47 \\
Women (\%) & 78 & 81 & 88 & 89 \\
Men (\%) & 22 & 19 & 12 & 11 \\
Average BMI $\left(\mathrm{kg} / \mathrm{m}^{2}\right)$ & 31,7 & 30,4 & 29,3 & 28,6 \\
High blood pressure $(\%)$ & 77 & 60 & 67 & 57 \\
Average Lesquene & 12,5 & 11 & 11 & 10,5 \\
WOMAC pain & 14 & 12 & 12 & 10 \\
WOMAC stiffness & 2 & 1,5 & 1 & 1 \\
WOMAC dysfunction & 58 & 49 & 46 & 44 \\
\hline
\end{tabular}

Conclusion: The epidemiological characteristics as well as the knee osteoar thritis characteristics are statistically close in the different groups of stages of risk of fall according to Tinetti test. Indeed, according to this study, knee osteoarthritis does not represent a significant risk factor for falls.

\section{REFERENCES:}

[1] Mat S, Tan MP, Kamaruzzaman SB, Ng CT. Physical therapies for improving balance and reducing falls risk in osteoarthritis of the knee: a systematic review. Age Ageing. 2015 Jan;44(1):16-24. doi: 10.1093/ageing/afu112. Epub 2014 Aug 22. PMID: 25149678.

[2] Köpke S, Meyer G. The Tinetti test: Babylon in geriatric assessment. Z Gerontol Geriatr. 2006 Aug;39(4):288-91. doi: 10.1007/s00391-006-0398-y. PMID: 16900448

Disclosure of Interests: None declared.

DOI: 10.1136/annrheumdis-2021-eular.1540

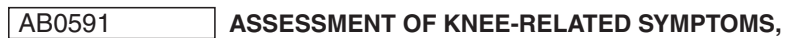 ACTIVITIES, AND QUALITY OF LIFE IN PATIENTS WITH POLYCYSTIC OVARY SYNDROME}

P. Kabakchieva ${ }^{1,2}$, T. Georgiev ${ }^{3,4}$, A. Gateva ${ }^{1,2}$, Z. Kamenov ${ }^{1,2} .{ }^{1}$ Medical University - Sofia, Department of Internal Medicine, Sofia, Bulgaria; ${ }^{2}$ Aleksandrovska University Hospital, Clinic of Endocrinology, Sofia, Bulgaria; ${ }^{3}$ Medical University - Varna, First Department of Internal Medicine, Varna, Bulgaria; ${ }^{4}$ St. Marina's Hospital, Rheumatology Clinic, Varna, Bulgaria

Background: Osteoarthritis $(\mathrm{OA})$ is a slowly progressive disease that probably begins in young adulthood when a timely treatment would present the possibility to reverse or slow down the disease process. Obesity and sex hormones play an essential role in the pathogenesis of $O A$ in women and their influence on joint function persists throughout the whole life [1]. Polycystic ovary syndrome (PCOS) is the most common endocrine disorder among women of reproductive age characterized by sex-hormonal disbalance, metabolic abnormalities, and most commonly obesity. It seems that PCOS presents the best opportunity to study the complex interactions among hormonal disbalance, obesity, and (pre) osteoarthritis.

Objectives: Our study aims to assess the knee-related symptoms, activities, and quality of life in young women with PCOS and to compare them with healthy volunteers using Knee Injury and Osteoarthritis Outcome Score (KOOS) developed for younger and more active individuals [2].

Methods: Fifty-four patients with PCOS who met the Rotterdam criteria were compared with 26 healthy women. Both groups were matched by age and body mass index (BMI). The exclusion criteria of the study were: the presence of inflammatory/autoimmune rheumatic disease and/or another endocrine disorder. Pregnant women and participants, who used systemic corticosteroids, antiandrogens, or insulin-sensitive drugs in the last 3 months were also excluded from the study. After written informed consent all participants filled in the questionnaire. KOOS was scored in each of its 5 domains: pain symptoms, activities of daily living (ADL), sport and recreation function, and knee-related quality of life (QoL). Additionally, detailed anthropometric data, clinical examination, and hormonal assessment (testosterone, dehydroepiandrosterone sulfate [DHEAS], androstenedione, 17-OH-progesterone, luteinizing hormone [ $\mathrm{LH}]$, follicle stimulating hormone [FSH], and estradiol) were carried out in all overnight-fasted participants during a follicular phase of a menstrual cycle. 
Results: Patients and healthy volunteers had similar anthropometric and demographic characteristics but PCOS women were expectedly more hyperandrogenic and hirsute than controls. Patients and controls did not differ significantly in their knee pain, knee-related QoL, and sport and recreation function. Knee-related symptoms were more prominent in the PCOS group compared to controls $(p=$ 0.035). ADL associated with the knee joint were also more impaired in patients $(p=0.001)$. Obese PCOS women had a significantly lower score in the ADL domain than normal and overweight patients $(p=0.035)$. In the PCOS group, both knee-related ADL and sport and recreation function correlated significantly with weight $(p=0.025$ and $p=0.034$, respectively) and waist circumference $(p=$ 0.011 and $p=0.016$, respectively), among all studied anthropometric parameters. Sex hormones did not correlate with any of the evaluated KOOS subscales. Conclusion: PCOS patients may experience impaired physical function related to daily life due to knee problems. Obesity may further contribute to knee-related ADL dysfunction. Hormonal disturbances did not show any association with knee complaints in our study.

\section{REFERENCES:}

[1] Georgiev T, Angelov AK. Modifiable risk factors in knee osteoarthritis: treatment implications. Rheumatol Int. 2019 Jul;39(7):1145-1157

[2] Roos EM, Toksvig-Larsen S. Knee injury and Osteoarthritis Outcome Score (KOOS) - validation and comparison to the WOMAC in total knee replacement. Health Qual Life Outcomes 2003;1:17.

Acknowledgements: The study was performed with the financial support of Medical University-Sofia, Bulgaria, Young Investigator 2020, Project No 8378/20.11.2019, Contract D-85/24.06.2020.

Disclosure of Interests: None declared.

DOI: 10.1136/annrheumdis-2021-eular.2072

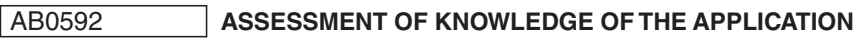 OF TOPICAL ANALGESICS}

S. Miladi ${ }^{1}$, W. Belhaj ${ }^{1}$, A. Fazaa ${ }^{1}$, M. Sellami ${ }^{1}$, K. Ouenniche ${ }^{1}$, L. Souebni ${ }^{1}$, S. Kassab ${ }^{1}$, S. Chekili ${ }^{1}$, K. Ben Abdelghani ${ }^{1}$, A. Laatar ${ }^{1} .{ }^{1}$ University of Tunis el Manar, Faculty of Medicine of Tunis, Mongi slim Hospital, Rheumatology Department, Tunis, Tunisia

Background: In chronic osteoarticular pain, especially knee osteoarthritis, topical analgesics and topical non-steroidal anti-inflammatory drugs represent an interesting therapeutic alternative for patients with several comorbidities in order to avoid side effects and medical interactions.

Objectives: The aim of this work was to assess how patients apply a topical analgesic.

Methods: A prospective study has been conducted over a period of 4 weeks. Sixty-six patients followed for knee osteoarthritis at different stages of the disease and under topical analgesic treatment were integrated into this study. Epidemiological data, physical examination and x-ray data were collected. The impact of knee osteoarthritis was evaluated by the Lequesne and WOMAC indices. Patients were asked about how long they apply the treatment, how often they use the treatment per week and whether they are massaging the aching area while applying the treatment.

Results: In the studied population, the majority of patients were women (87\%) with an average age of 55 years. Forty percent of the patients were illiterate as long as $21 \%$ received a college education. Patients' occupations were distributed as follows: $59 \%$ housewives, $21 \%$ retirees, $12 \%$ manual occupations and $8 \%$ non-manual occupations.

The most common comorbidities were high blood pressure $(61 \%)$, diabetes $(38 \%)$ and osteoporosis (36\%).

The average duration of development of osteoarthritis of the knee was 9 years. The most common radiological stage was stage 1 (58\%). The site was femorotibial in $88 \%$ of cases, patellofemoral in $4 \%$ of cases and bi or tri-compartmental in $8 \%$ of cases. The osteoarthritis most often affected both knees: $86 \%$ of cases The average of the Lequesne index was 11. The average WOMAC index was 12 for pain, 1.5 for stiffness and 48 for dysfunction.

All the interviewed patients were on topical analgesics for an average of 5 years. The average frequency of application of topical analgesics was 9 times per week. Housewives and retirees used the treatment on average 14 times per week while working patients use the treatment on average 5 times per week. Seventy-two percent of patients reported that they performed an average massage lasting 11 minutes with each application.

Among patients with secondary and university education, $64 \%$ of patients reported that they performed a massage on the painful knee while applying the medication.

Patients followed for knee osteoarthritis for more than 10 years tend to apply the local analgesic for a shorter period of time (an average of 5 minutes).

Conclusion: Patients followed for osteoarthritis of the knee who are taking topical analgesics tend to make misuse, including massaging for a long time and applying treatment less frequently than recommended. The level of education does not seem to play a role in knowing the right way to apply. However, patients who have been followed for a longer period for knee osteoarthritis use the treatment more correctly.

\section{REFERENCES:}

[1] Flores MP, Castro AP, Nascimento Jdos S. Topical analgesics. Rev Bras Anestesiol. 2012 Mar-Apr;62(2):244-52. doi: 10.1016/S0034-7094(12)701228. PMID: 22440379.

[2] Barkin RL. The pharmacology of topical analgesics. Postgrad Med. 2013 Jul;125(4 Suppl 1):7-18. doi: 10.1080/00325481.2013.1110566911. PMID: 24547599.

Disclosure of Interests: None declared.

DOI: 10.1136/annrheumdis-2021-eular.2148

\section{$\mathrm{AB} 0593$ \\ PERCEPTION OF THE EFFECTIVENESS OF TOPICAL ANALGESICS IN PATIENTS WITH OSTEOARTHRITIS} OF THE KNEE

W. Belhaj ${ }^{1}$, S. Miladi ${ }^{1}$, A. Fazaa ${ }^{1}$, M. Sellami ${ }^{1}$, K. Ouenniche $^{1}$, L. Souebni $^{1}$, S. Kassab ${ }^{1}$, S. Chekili ${ }^{1}$, K. Ben Abdelghani ${ }^{1}$, A. Laatar ${ }^{1} .{ }^{1}$ University of Tunis el Manar, Faculty of Medicine of Tunis, Mongi slim Hospital, Rheumatology Department, Tunis, Tunisia

Background: Osteoarthritis of the knee is one of the most common joint diseases. It mainly manifests as pain and can become disabling over time. Several symptomatic therapeutic methods are used to relieve patients with knee osteoarthritis and give them more autonomy. Topical analgesics are considered as an interesting alternative for old and poly-medicated patients.

Objectives: The aim of this work was to assess the perception of the effectiveness of topical analgesics by patients followed for knee osteoarthritis.

Methods: A prospective study has been conducted collecting 66 patients followed for knee osteoarthritis at different stages of the disease and under topical analgesic treatment. Epidemiological data, physical examination and $\mathrm{x}$-ray data were collected. The impact of knee osteoarthritis was evaluated by the Lequesne and WOMAC indices. Patients were asked to evaluate their perception about: the time that makes the medication to act, the duration of action and overall satisfaction with the topical analgesic by rating it from 0 to 5 . Zero represents complete dissatisfaction while 5 represents complete satisfaction. Patients were also asked about the side effects observed after the use of the topical treatment and whether they recommended this treatment to a friend or a parent.

Results: In the studied population, the majority of patients were women (87\%) with an average age of 55 years. The most common comorbidities were high blood pressure $(61 \%)$, diabetes (38\%) and osteoporosis (36\%). Sixty-nine percent of patients were taking more than two oral treatments. The average duration of development of osteoarthritis of the knee was 9 years. The most common radiological stage was stage $1(58 \%)$. The site was femorotibial in $88 \%$ of cases, patellofemoral in $4 \%$ of cases and bi or tri compartmental in $8 \%$ of cases. The osteoarthritis most often affected both knees in $86 \%$ of cases.

The average of the Lequesne index was 11. The average WOMAC index was 12 for pain, 1.5 for stiffness and 48 for dysfunction.

All the patients surveyed were on topical non-steroidal anti-inflammatory drugs (NSAIDs), 37.7\% combined local herbal medicine with local NSAIDs, $53 \%$ were taking oral analgesics, and $19 \%$ were using oral non-steroidal anti-inflammatory drugs in the past month.

The average rating for time that takes the topical treatment to act was 2.9 , for duration of action was 1.2 , and for overall satisfaction was 2.8 . No adverse effects of topical treatment have been reported.

For patients over 60 years old, better satisfaction was noted (the average score for overall satisfaction was 3.2)

Patients with a WOMAC Pain Index greater than 10 were less satisfied (the average overall satisfaction score was 1.1). Patients who took both topical NSAIDs and topical herbal medicine reported that topical NSAIDs were more effective in $66 \%$ of cases. Forty percent of patients recommended topical treatment to a friend or a family member.

Conclusion: In patients with chronic pain associated to osteoarthritis of the knee, topical analgesics are considered a good long-term therapeutic alternative. Patients are generally satisfied with the effectiveness of the local treatment, in particular the immediate effect felt after application, but less satisfied with the duration of action. Perception of effectiveness appears to be affected by age and degree of pain.

\section{REFERENCES:}

[1] D. Y. Wall, "Topical Nonsteroidal Anti-Inflammatory Drugs for Chronic Musculoskeletal Pain in Adults," p. 3, 2017

[2] R. L. Barkin, "The Pharmacology of Topical Analgesics," null, vol. 125, no. sup1, pp. 7-18, Jul. 2013, doi: 10.1080/00325481.2013.1110566911.

Disclosure of Interests: None declared.

DOI: 10.1136/annrheumdis-2021-eular.2164 